\title{
Politique
}

Politique

\section{René Dumont, Finis les lendemains qui chantent... La Chine décollectiviste, Paris, Seuil, 1984, 338 p.}

\section{Rodolphe De Koninck}

Numéro 7, hiver 1985

Projection internationale du Québec

URI : https://id.erudit.org/iderudit/040488ar

DOI : https://doi.org/10.7202/040488ar

Aller au sommaire du numéro

Éditeur(s)

Société québécoise de science politique

ISSN

0711-608X (imprimé)

1918-6584 (numérique)

Découvrir la revue

Citer ce compte rendu

De Koninck, R. (1985). Compte rendu de [René Dumont, Finis les lendemains qui chantent... La Chine décollectiviste, Paris, Seuil, 1984, 338 p.] Politique, (7),

143-148. https://doi.org/10.7202/040488ar d'utilisation que vous pouvez consulter en ligne.

https://apropos.erudit.org/fr/usagers/politique-dutilisation/ 
RECENSIONS

René Dumont, Finis les lendemains qui chantent... La Chine décollectivise, Paris, Seuil, 1984, 338 p.

Comme plusieurs des livres de René Dumont, celui-ci a été écrit très rapidement et possède donc les habitueles qualités et 
défauts. On y trouve la manifestation d'une grande curiosité combinée avec une incapacité à présenter les données d'une manière rigoureuse et systématique; une grande érudition agronomique qui a tendance, quelquefois, à se disperser; une capacité de synthèse versant, quelquefois, dans la simplification ou la confusion; une irréductible naïveté politique basculant, quelquefois, dans le cynisme.

Ceci dit, Dumont possède une bonne connaissance de la Chine qu'il a visitée à plusieurs reprises depuis 1929, visites qu'il cite en introduction et dont il a tiré des enseignements mis à profit dans la première partie du livre. L'auteur y relate ce qu'il appelle «la longue marche du peuple chinois», en particulier depuis 1949. Après avoir brièvement évoqué les vicissitudes de l'histoire de la paysannerie chinoise au cours des siècles ayant précédé la prise du pouvoir communiste, il s'attarde aux nombreuses réformes qui, particulièrement dans les campagnes, se sont succédées depuis la fondation de la République Populaire de Chine. La réforme agraire proprement dite, réalisée de 1949 à 1957, devait elle-même passer par plusieurs étapes. Alors qu'en 1950, la distribution des terres aux quelque 120 millions de familles paysannes avait été accélérées, elle fut brutalement interrompue dès 1953. C'est alors que débuta la formation des coopératives; la petite paysannerie chinoise qui avait attendu pendant longtemps un meilleur accès au faire-valoir direct se voyait à nouveau mobilisée pour un projet orchestré d'en haut, par l'État. En 1958, la collectivisation franchit une étape cruciale: les communes populaires sont créées. Au cours des sept années qui vont suivre, dans les campagnes de Chine, se feront d'abord sentir les effets de ce que Dumont appelle le «Grand Bond en arrière", suivi d'une brève période de redressement. En effet autant le soit-disant Grand Bond en avant allait-il s'avérer un échec dans le domaine industriel, 
autant dans les communes populaires la production agricole allaitelle rencontrer de sé.rieux problèmes. Dans bien des cas, de 1958 à 1961, la production s'effondre; puis, comme Dumont l'indique, de 1962 à 1966, on panse les plaies. Pour y parvenir, il faut renverser la tendance au gigantisme, lequel est de toute évidence d'inspiration soviétique. C'est donc dès cette époque que furent initiées certaines formes de décentralisation, aujourd'hui beaucoup plus généralisée. L'unité de compte ne relèvera plus de la commune, ni même de la brigade mais bien de l'équipe. On rétablit, en 1961-62, toute une série d'activités privées, notamment l'exploitation des lopins individuels qui vont couvrir $5 \%$ à $7 \%$ des terres arables. En 1962, dans le Guangdong et l'Anhui, «on remet à l'honneur ... des contrats familiaux..., déjà essayés dès 195657» (p. 67). Cette évolution n'est pas uniforme, ni sans retour en arrière. Ainsi, en 1964, Mao ordonne la suppression des lopins individuels. Mais au total l'agriculture a progressé et, en 1966, le niveau de production absolu de 1957 est retrouvé.

Puis en 1966, survient la Révolution Culturelle. La primauté absolue du politique va bouleverser la Chine, d'abord urbaine puis rurale. Dumont en parle peu, et pour cause: la documentation sur le déroulement et les effets de cette décennie (1966-1976) de chaos économique, qui va laisser des traces encore profondes aujourd'hui, est complexe, incomplète et souvent contradictoire. La consulter et l'utiliser avec sagesse est une affaire de patience et d'érudition, dont Dumont ne peut s'embarrasser. Il dresse, avec un peu plus de rigueur, les caractéristiques des changements survenus dans la politique agricole depuis 1977. Les plus importants relèvent assurément de ces «nouveaux systèmes de responsabiité», approuvés en décembre 1978 à l'occasion du $\mathrm{III}^{\mathrm{e}}$ plénum du $\mathrm{XI}^{\mathrm{e}}$ comité central. Leur mise en place fut amorcée dès 1979, accélérée en 1981 et 1982; ils touchent aujourd'hui la quasi-totalité des paysans chinois. Cette nouvelle politique agricole représente une véritable réhabilitation de la famille comme unité de travail. Même si la 
gestion d'une bonne partie des moyens de production demeure collective, le travail agricole fait bel et bien l'objet d'une décollectivisation. Ainsi, non seulement chaque famille dispose-t-elle d'un lopin individuel, mais elle passe un contrat avec l'équipe, qui pour la culture d'une parcelle appartenant à l'État, qui pour l'élevage des porcs, celui des canards, etc. Bref, les paysans chinois sont devenus des fermiers de l'État. Comme le souligne Dumont, il ne s'agit pas de métayage, mais bien de fermage. L'État demeure propriétaire du fond de terre, mais les forfaits - généralement fixés en nature - auxquels souscrivent les paysans leur permettent de tirer eux-mêmes profit de tout accroissement de rendement réalisé sur les parcelles dont ils ont la responsabilité. La plus grande initiative ainsi laissée aux paysans contribue à un accroissement substantiel de la production agricole, relève le revenu et le niveau de vie des ruraux, réduit l'écart économique entre la ville et la campagne mais contribue également à une polarisation sociale au sein même des communautés agricoles. Dumont évoque aussi, mais documente peu, une montée des «nouveaux riches».

La deuxième partie du livre contient un examen plus détaillé du déroulement et des effets de cette véritable réforme agraire dans plusieurs régions et provinces de la Chine. Tour à tour, sont abordés l'Anhui, le Sichuan, le Yunnan, le Guangdong, la municipalité de Shanghai, le Shaanxi et le Shangdong. On le voit, le survol est large. Même si ni les provinces de Mandchourie, ni les régions autonomes de l'ouest du pays ne sont évoquées, le tableau dressé est plus représentatif que celui que l'on trouve dans bien des livres sur la Chine alors que seules une ou deux régions sont vraiment traitées. Certes, la méthode de Dumont manque de rigueur: des rappels historiques chevauchent des descriptions contemporaines; les anecdotes, les bilans d'auteurs plus ou moins correctement cités, les analyses et intuitions agronomiques de l'auteur, souvent remarquables, tout cela est livré pêle-mêle; les statistiques sont nombreuses, leur origine est souvent obscure, 
elles apparaissent ici et là à travers le texte même et ne sont jamais regroupées sous forme de tableau. Malgré cela, malgré ce manque de rigueur évident, le développement de la démonstration se réalise. Dumont maintient son cap, ce qui n'est pas un mince exploit à travers ce qui peut paraître confus. Il en ressort l'image d'une Chine rurale en plein bouleversement, une Chine fort diverse, beaucoup moins monolithique qu'on a tendance à le croire, une Chine où les paysans, à défaut d'avoir accès au pouvoir politique qu'ils ont contribué à remettre au Parti au moment de la révolution, semblent gagner du terrain sur le plan économique.

Cette dialectique entre pouvoir et initiative économique peut être considérée comme le principal objet de la troisième partie du livre alors que l'écriture de Dumont devient plus débridée et où les sujets abordés plus dispersés. Tout en se réjouissant devant la déstalinisation et la démaoïsation et donc devant l'amélioration ainsi assurée à l'agriculture face à l'industrie, Dumont rappelle combien les revirements sont toujours possibles. Les inégalités, les privilèges, la Nomenklatura sont évoqués. L'actuelle politique démographique est discutée, notamment l'accentuation de l'infanticide des filles qu'elle entraîne. Puis, dans les deux derniers chapitres, pour faire bonne mesure, Dumont passe à l'Inde, au Bangla Desh et effectue un bref retour à la Chine et aux problèmes des despotismes dans le Tiers Monde.

Malgré le manque de rigueur qui ici encore caractérise les envolées de l'auteur, on doit lui reconnaître un grand mérite: il ne peut laisser son lecteur indifférent. Malgré la dispersion de sa curiosité et de ses connaissances, malgré cette naïveté qui lui fait citer certaines statistiques, de toute évidence fausses - notamment concernant les rendements agricoles dans la région de Shanghai - qu'il avait déjà consignées dans Chine, la révolution culturale (livre publié dans la même collection en 1976), malgré sa boulimie intellectuelle parfois déroutante et cette formidalbe énergie physique 
qu'il sait mettre à son service, malgré et à cause de tout cela, l'œuvre de Dumont est décapante. Elle révèle des mouvements sociaux majeurs, elle met à jour des grands problèmes, elle les éclaire et les critique. Dans le cas de la Chine et plus particulièrement de la paysannerie chinoise, l'œuvre de Dumont ne saurait avoir la profondeur et l'originalité de celles d'un W. Skinner ou d'un W. Hinton, mais elle est utile, ne serait-ce que par son dynamisme, ses revirements, ses contradictions, toutes choses dont témoigne ce livre.

Rodolphe De Koninck

Université Laval 